\title{
Reliability study of the Pectoralis Minor Muscle Thickness Measurement using Rehabilitative Ultrasound Imaging
}

\author{
Ji Young Lim, PT, MS • Se-Yeong Lee, $P T^{1} \cdot$ Seung-Hwa Jung, PT, MS • Dae-Sung Park, PT, PhD ${ }^{2 \dagger}$
}

Department of Physical Therapy, Graduate School of Medical Science, Konyang University,

${ }^{1}$ Department of Physical Therapy, Daejeon Yuseong Sun Hospital,

${ }^{2}$ Department of Physical Therapy, Konyang University

Received: April 04, 2021 / Revised: April 15, 2021 / Accepted: May 06, 2021

(c) 2021 J Korean Soc Phys Med

\section{| Abstract |}

PURPOSE: This study examined the imaging procedure of pectoralis minor muscle thickness and assessed the intra- and inter-rater reliability of the muscle thickness measured by two raters using rehabilitative ultrasound imaging (RUSI) in healthy individuals.

METHODS: Fifteen participants (aged 21 - 28, seven females, and eight males) were involved in the study. The primary rater palpated the coracoid process and the fourth rib, defined as the width of the index finger lateral to the sternum to avoid breast tissues, and lined the two landmarks. The second examiner checked $1 / 3$ (1st point) and $1 / 2$ (2nd point) of the line length as measurement points. The two raters obtained right side muscle images of the participants at a standardized sitting position using RUSI with a $7.5 \mathrm{MHz}$ linear transducer at $40 \mathrm{~mm}$ depth. For intra-rater reliability, the principal rater took three images per point and tried to take one more with an interval. For the inter-rater reliability, the other rater performed the same tasks as the principal rater on the

†Corresponding Author : Dae-Sung Park daeric@konyang.ac.kr, https://orcid.org/0000-0003-4258-0878

This is an Open Access article distributed under the terms of the Creative Commons Attribution Non-Commercial License (http://creativecommons.org/licenses/by-nc/3.0) which permits unrestricted non-commercial use, distribution, and reproduction in any medium, provided the original work is properly cited. same day. The reliability was analyzed using the intra-class correlation coefficient (ICC), the standard error of the measurement (SEM), and Bland and Altman plots.

RESULTS: The reliability at all points was excellent for the same rater $\left(\mathrm{ICC}_{3,1}=.973-.978, \mathrm{SEM}=.042-.046\right)$, and between raters $\left(\mathrm{ICC}_{2,1}=.939-.959, \mathrm{SEM}=.059-.097\right)$. CONCLUSION: These findings show that the RUSI could be reliable for examining the pectoralis minor muscle thickness in healthy individuals at all measurement sites.

Key Words: Pectoralis muscles, Rehabilitative ultrasound imaging, Reproducibility of the results

\section{Introduction}

During arm elevation, the pectoralis minor, the only scapulothoracic muscle, works as an antagonist to scapular motion [1-4]. Shortening or tightness of the pectoralis minor muscle can be a possible consequence of increased protraction and anterior tipping in the long-term [5,6]. The tightness of the pectoralis minor, which attaches the coracoid process of the scapula and at the third, fourth, and fifth ribs near their sternocostal junctions, has been described as a possible factor in shoulder impingement syndrome $[2,3]$. 
Reliability and validity studies in muscle morphology measurements using rehabilitative ultrasound imaging (RUSI) have been investigated actively [7,8]. This clinical measurement technique is safe from radiation exposure, easy to handle, and noninvasive. In physical therapy, RUSI could be used to provide biofeedback during specific muscle contractions for muscle rehabilitation and re-education, especially in the case of a deep muscle (i.e., transverse abdominis) $[9,10]$. Furthermore, it could be useful for evaluating the muscle morphology and behavior, such as the change in muscle thickness and cross-sectional area during exercise and static or dynamic physical tasks $[10,11]$. RUSI can assist in therapeutic interventions in physical therapy [11]

Previous studies provided reliable results for measuring the muscle thickness of the serratus anterior muscle $[12,13]$; rhomboid major [14], middle [15], and lower trapezius [16]; supraspinatus [17,18]; and infraspinatus [19]. Although the reliability of RUSI measurements for several shoulder muscle thicknesses has been demonstrated, the reliability of small pectoral muscle thickness measurements by RUSI has not yet been established in healthy adults without a shoulder pathology.

The representative measurement technique considered the origin at the $3 \mathrm{rd}$, 4th, and 5th rib and insertion at the coracoid process of pectoralis minor muscle. The pectoralis minor length measurements in healthy subjects without a history of shoulder pathology were validated using a tape or calipers [20].

This study examined the measurement points of the pectoralis minor muscle by RUSI and focused on its measurement technique description, which is referred to as a measurement technique validated by Borstad and Ludewig [3] on human cadavers, and by Borstad [21] both in vitro and in vivo. The intra- and inter-rater reliability of the measurement at two points of the pectoralis minor muscle was examined using RUSI.

\section{Methods}

\section{Participants}

Fifteen healthy participants (aged 21-28, seven females, and eight males) were enrolled in this study by convenience sampling from students in the department of physical therapy of Konyang University. The inclusion criteria were asymptomatic adults who did not have a musculoskeletal history, such as shoulder surgery, fracture, and dislocation. Participants who have a limited range of motion of shoulder and chest muscle pain were excluded. The Institute Review Board of the Konyang University approved all procedures of this study (No. KYU-2018-079-01). All subjects signed an informed consent form for the participants.

\section{Experimental procedure}

1) Raters

Two raters carried out the imaging procedure. The primary rater (rater A) of a master's degree experienced the RUSI. The other rater (rater B) was a novice in RUSI who had practiced imaging the pectoralis minor muscle before beginning the study.

\section{2) RUSI procedure}

The raters obtained muscle images using a real-time ultrasound scanner in B-mode with a $7.5 \mathrm{MHz}$ linear transducer (MySono U6, Samsung Medison, South Korea). All images were a uniform $40 \mathrm{~mm}$ in depth. Before the measurements started, the participants sat in the standardized position on a chair with a backrest, flexing the hip and knee $90^{\circ}$, pronating their forearm, and putting their hands on the thigh. The participants took a break if they felt fatigued.

A protocol was performed in the following manner. First, rater A palpated the coracoid process, and the $4^{\text {th }}$ rib, which is defined as the width of the index finger lateral to the sternum to avoid breast tissues, lined with two landmarks. 


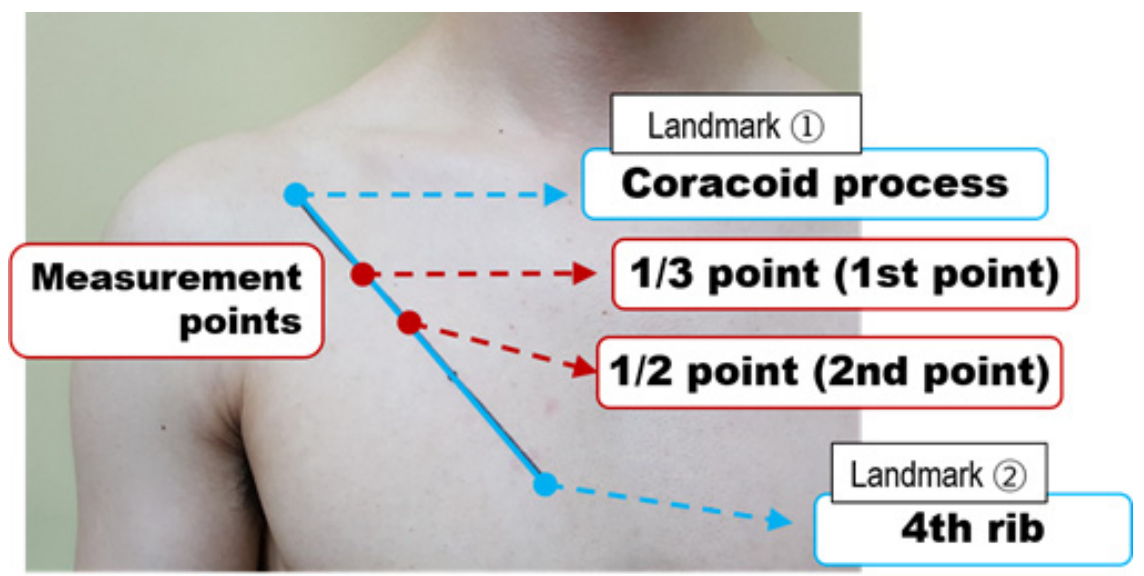

Fig. 1. 1/3 (1st point) and $1 / 2$ (2nd point) of the line length as the measurement points.
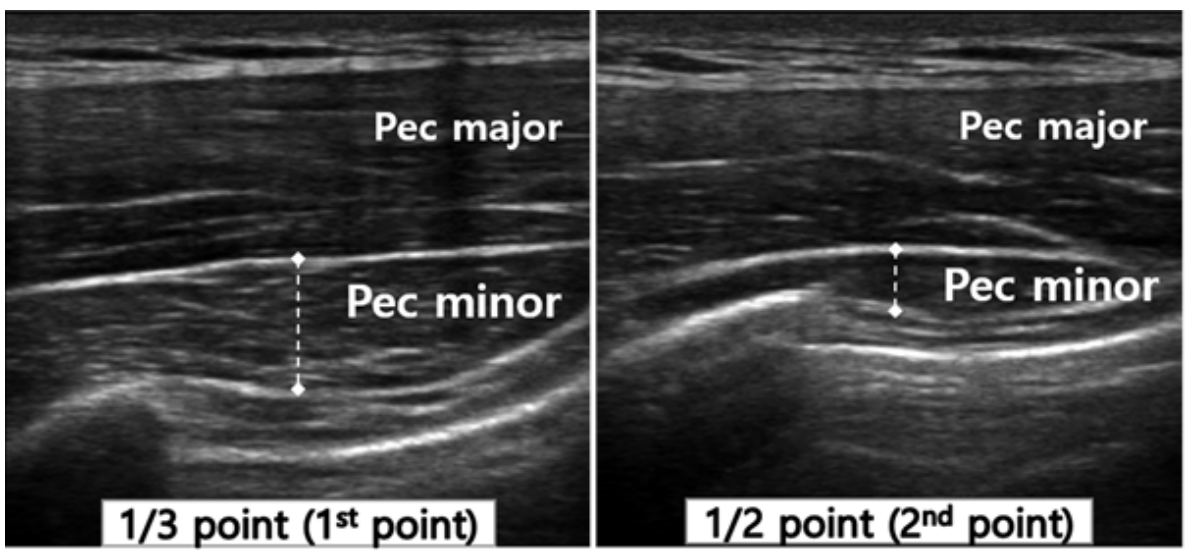

Fig. 2. Pectoralis minor muscle thickness was measured at the middle point of the ultrasound image in the $1 / 3$ point (left) and $1 / 2$ point (right).

The second, rater A marked $1 / 3$ (1st point) and $1 / 2$ (2nd point) of the line length as measurement points with a permanent marker (Fig. 1). The raters put the probe on the transverse axis of the pectoralis minor muscle at the resting state and obtained images at the end-expiration to minimize the effects of breathing and muscle contraction. Pectoralis minor muscle images were obtained on the right side.

For intra-rater reliability, rater A took three images per measurement point and tried one more with a 30-minute interval. For the inter-rater reliability, rater B performed the same task as rater A on the same day. After all images of the subjects were collected, the muscle thickness was measured. The raters did not know the muscle thickness of each subject while collecting the RUSI image.

\section{Measurements}

After collecting all images, the muscle thickness was measured at the middle point of the image $(4 \mathrm{~cm}$ width and $4 \mathrm{~cm}$ height) using Sonoview (MySono U6, Samsung Medison, South of Korea) by rater A. The cursor was placed on the inside edge of the facial borders of the muscle and drawn vertically at the top of the fascial borders (Fig. 2). 


\section{Data analysis}

Both intra-rater and inter-rater reliability were analyzed using the intra-class correlation coefficients (ICC) as the relative reliability and the standard error of the measurement (SEM) as the absolute reliability. The model and type of ICC followed a flowchart for the selection procedure of the ICC form in a previous study [22]. The $\mathrm{ICC}_{3,1}$ and $\mathrm{ICC}_{2,1}$ models were used to evaluate the intra-rater reliability and inter-rater reliability, respectively. For ICC interpretation, the threshold of the ICC was suggested; values less than $.5, .5-.75, .75-.90$, and .90 - 1.00 were considered poor, moderate, good, and excellent reliability, respectively. The $95 \%$ confidence interval $(95 \%$ CI) was calculated for each measured point to provide a visual distribution of the muscle thickness [23]. The Bland and Altman plot indicated visually whether the values were within the degree of agreement (mean $\pm 2 \mathrm{SD}$ ) between tests 1 and 2 by rater $A$ and between the two raters who performed the same procedure [23].

\section{Results}

Table 1 lists the demographic characteristics of the participants. The pectoralis minor muscle thickness at the 1st point obtained by rater A was $.91 \pm .23 \mathrm{~mm}$ in the 1st test and $.91 \pm .24 \mathrm{~mm}$ in the $2^{\text {nd }}$ test. The thickness at the $2^{\text {nd }}$ point was $.51 \pm .24$ and $.53 \pm .29$, respectively (Table 1). The intra-rater reliability at both points was $\mathrm{ICC}_{3,1} .973-.978$ with $95 \% \mathrm{CI}(.875-.991)$ and the SEM was low ranging from .42 to .46 (Table 2).
Table 1. General Characteristic of the Participants $(N=15)$

\begin{tabular}{cc}
\hline Variable & Mean (SD) or number \\
\hline Gender: male / female (n) & $8 / 7$ \\
Age (years) & $23.73(1.98)$ \\
Height $(\mathrm{cm})$ & $165.93(8.61)$ \\
Weight $(\mathrm{kg})$ & $57.29(10.31)$ \\
Body Mass Index $\left(\mathrm{kg} / \mathrm{m}^{2}\right)$ & $20.63(2.05)$ \\
\hline
\end{tabular}

The pectoralis minor muscle thicknesses at the 1st point obtained by raters $\mathrm{A}$ and $\mathrm{B}$ were $.91 \pm .23 \mathrm{~mm}$ and .94 \pm .26 , respectively, and $.51 \pm .24$ and $.56 \pm .32$ respectively, at the $2^{\text {nd }}$ point (Table 2). The inter-rater reliability at two points was $\mathrm{ICC}_{2,1} .939-.959$ with the $95 \%$ CI $(.818$ .986). The SEM showed a low value ranging from .059 to .097 (Table 3).

The Bland and Altman's plots showed good agreement for the intra-rater reliability between the two measures sessions and inter-rater reliability in Fig. 3. The mean difference was close to zero $(.01-.05 \mathrm{~mm})$. There were outliners at all points in the intra-and inter-rater reliability.

\section{Discussion}

The current study evaluated whether the RUSI could reproduce the consistent thickness values of the pectoralis minor muscle at all points measured by both raters in healthy young individuals. To the best of the authors' knowledge, this was the first study that explored the imaging procedure for measuring the thickness of the pectoralis minor muscle using the RUSI. These findings

Table 2. Intra-rater Reliability for Pectoralis Minor Muscle Thickness Measurements

\begin{tabular}{cccccc}
\hline \multirow{2}{*}{$\begin{array}{c}\text { Measurement } \\
\text { points }\end{array}$} & \multicolumn{2}{c}{ MT $(\mathrm{cm})($ mean \pm SD) } & \multicolumn{3}{c}{ Reliability } \\
\cline { 2 - 7 } & 1st test & 2nd test & ICC $_{3,1}$ & $95 \%$ CI & SEM \\
\hline 1st point & $.91 \pm .23$ & $.91 \pm .24$ & .978 & $.875-.985$ & .042 \\
2nd point & $.51 \pm .24$ & $.53 \pm .29$ & .973 & $.919-.991$ & .046 \\
\hline
\end{tabular}

MT, muscle thickness; SD, standard deviation; ICC, intra-class correlation coefficient; $95 \%$ CI, 95\% confidence interval; SEM, standard error of the mean 
Table 3. Inter-rater Reliability for Pectoralis Minor Muscle Thickness Measurements

\begin{tabular}{cccccc}
\hline \multirow{2}{*}{$\begin{array}{c}\text { Measurement } \\
\text { points }\end{array}$} & \multicolumn{2}{c}{ MT $(\mathrm{cm})($ mean \pm SD) } & \multicolumn{3}{c}{ Reliability } \\
\cline { 2 - 6 } & Rater A & Rater B & ICC $_{2,1}$ & $95 \%$ CI & SEM \\
\hline 1st point & $.91 \pm .23$ & $.94 \pm .26$ & .959 & $.877-.986$ & .097 \\
2nd point & $.51 \pm .24$ & $.56 \pm .32$ & .939 & $.818-.979$ & .059 \\
\hline
\end{tabular}

MT, muscle thickness; SD, standard deviation; ICC, intra-class correlation coefficient; $95 \%$ CI, 95\% confidence interval; SEM, standard error of the mean

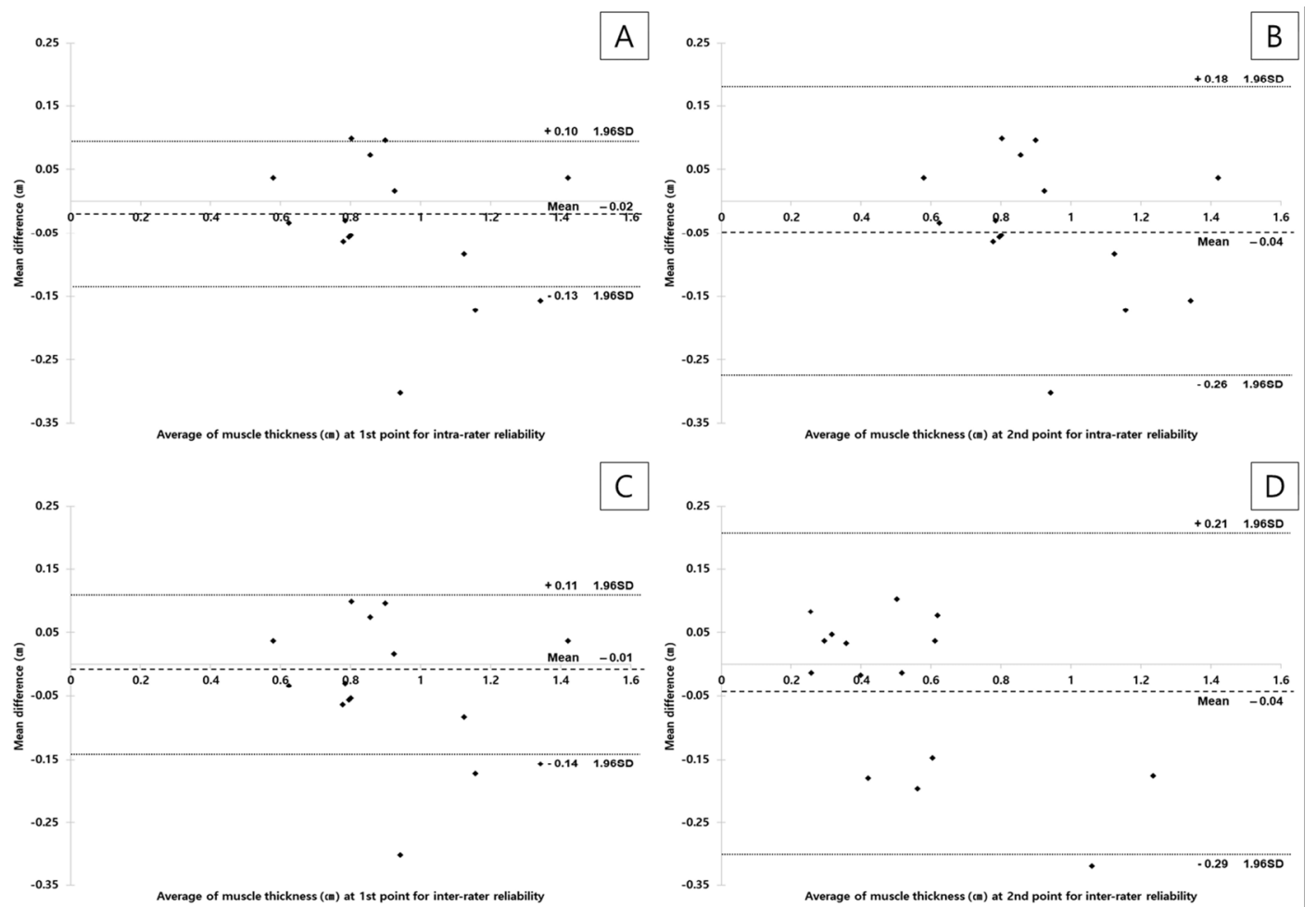

Fig. 3. Bland-Altman plots of the intra-rater reliability at 1st point $(A)$, and 2 nd point $(B)$ and inter-rater reliability at the 1st point $(C)$, and 2 nd point (D).

show that the use of RUSI for measuring the pectoralis minor muscle thickness had excellent intra- and inter-rater reliability with a narrow $95 \% \mathrm{CI}$. Moreover, the SEM of both measurement points was very small in the intra- and inter-rater reliability at both measurement sites. The SEM within $10 \%$ of the mean muscle thickness value was acceptable for measuring the muscle thickness of the pectoralis minor [24].
Based on an interpretation of the ICC value [22], the thickness measurement of the pectoralis minor muscle using RUSI had excellent intra- and inter-rater reliability at all measurement points. Similarly, the intra- and inter-rater reliability of the shoulder muscles thickness, which has a distinctive role concerning shoulder stabilization, were moderate to excellent in the middle trapezius (ICC $=.67$ - .81)[15], lower trapezius (ICC $=.91-.99$ and .88)[16], 
rhomboid major (ICC $=.93-.98$ and $.75-.94)[14]$, and serratus anterior $(\mathrm{ICC}=.87-.97$ and $.43-.54)[12,13]$. The higher intra- and inter-rater reliability in the pectoralis minor compared to ICCs values of the aforementioned studies were because of the use of the same skin markings for the transducer setting and measurements of the intra-rater reliability on the same day. The excellent ICCs, which were observed in the intra- and inter-rater reliability, resulted from the short measurement period and the same scanning sites used by the two raters.

The anatomical position of the pectoralis minor originates from the coracoid process and attaches to ribs 3,4 , and 5 . The fourth rib was used as a landmark based on a reference to a validity study that examined the length of the pectoralis minor muscle in cadaver and healthy subjects [20]. During the pilot experiment, the 1/3,1 / 2, and $2 / 3$ points of the muscle length were determined according to muscle fibers direction of the pectoralis minor. Although this study examined the $2 / 3$ point of length (proximal to the sternum and costal cartilage) using RUSI, clear muscle fascia could not be found because it was located at the left border of the picture or was too scare and small to measure the muscle thickness. Therefore, this study recommended the 1 st and 2 nd points as scanning sites, which had excellent intra- and inter-rater reliability for measuring the pectoralis minor muscle thickness using RUSI.

A previous study [25] reported the reliability of ultrasonography for the pectoralis major thickness measurements and explained several factors affecting the muscle thickness measurements as a study limitation. The pectoralis major muscle is located below the pectoralis major. Hence, the factors to consider when measuring muscle thickness may be similar. Furthermore, changes in the amount of pressure, contact area, or transducer angle are well-known factors that affect the muscle thickness measurements in RUSI [26,27]. In particular, in cases of the pectoralis region, excessive chest expansion and constriction during breathing could lead to changes in thoracic curvature and variations of the muscle tension [25]. This means that physical therapists using RUSI for measuring the pectoralis minor muscle thickness need to pay more attention to the pressure of the probe and breathing instructions.

This study supports the method measuring the pectoralis minor muscle using RUSI in healthy populations to compare with pathological populations in a subsequent study. For a future study, researchers should substantiate the reliability of this procedure in people with shoulder pain, round shoulders, or thoracic outlet syndrome and investigate the function of RUSI in shoulder rehabilitation. Furthermore, the relationship between the pectoralis minor muscle thickness measured using RUSI and the pectoralis minor length measured using other methods needs to be determined to evaluate muscle shortening due to posture.

\section{Conclusion}

The current study established excellent reliability for the same rater at all measurement points of the pectoralis minor muscle using rehabilitative ultrasound imaging. This imaging procedure described in this study had high reproducibility between the two raters with experience of RUSI. For excellent reliability, different examiners need to be careful with breathing and pressure when examining the pectoralis minor muscle using RUSI. Overall, RUSI can be used to examine the pectoralis minor muscle thickness.

\section{Acknowledgments}

The authors wish to thank Professor Chang-Hyung Lee of Pusan National University Yangsan Hospital for helping with the ultrasound imaging. 


\section{References}

[1] Taylor DC, Dalton JR JD, Seaber AV, et al. Viscoelastic properties of muscle-tendon units: the biomechanical effects of stretching. Am J Sports Med. 1990;18(3):300-9.

[2] Ludewig PM, Cook TM. Alterations in shoulder kinematics and associated muscle activity in people with symptoms of shoulder impingement. Phys Ther. 2000;80(3):276-91.

[3] Borstad JD, Ludewig PM. The effect of long versus short pectoralis minor resting length on scapular kinematics in healthy individuals. J Orthop Sports Phys Ther. 2005;35(4):227-38.

[4] Phadke V, Camargo P, Ludewig P. Scapular and rotator cuff muscle activity during arm elevation: a review of normal function and alterations with shoulder impingement. Braz J Phys Ther. 2009;13(1):1-9.

[5] Kendall FP, McCreary EK, Provance PG, et al. Muscles, testing and function: with posture and pain (4th ed). London. Williams \& Wilkins Baltimore, MD. 1993.

[6] Sahrmann S. Diagnosis and treatment of movement impairment syndromes. United Statesof America. Elsevier Health Sciences. 2002.

[7] Fernández Carnero S, Arias Buria JL, Cuenca Zaldivar $\mathrm{JN}$, et al. Rehabilitative ultrasound imaging evaluation in physiotherapy: piloting a systematic review. Appl Sci. 2019;9(1):181.

[8] Dupont AC, Sauerbrei EE, Fenton PV, et al. Realtime sonography to estimate muscle thickness: Comparison with MRI and CT. J Clin Ultrasound. 2001;29(4):230-6.

[9] Hides J, Richardson C, Jull G, et al. Ultrasound imaging in rehabilitation. Aust J Physiother. 1995;41(3):187-93.

[10] Fernández-Carnero S, Calvo-Lobo C, Garrido-Marin A, et al. 2nd Rehabilitative Ultrasound Imaging Symposium in Physical Therapy, Madrid, Spain, 3-5 June 2016. Br J Sports Med. 2018;52:A1-A4.

[11] Teyhen D. Rehabilitative Ultrasound Imaging Symposium, May 8-10, 2006, San Antonio, Texas. J Orthop Sports
Phys Ther. 2006;36(8):A-1-A-17.

[12] Talbott NR, Witt DW. Ultrasound imaging of the serratus anterior muscle at rest and during contraction. Clin Physiol Funct Imaging. 2013;33(3):192-200.

[13] Talbott NR, Witt DW. Ultrasound examination of the serratus anterior during scapular protraction in asymptomatic individuals: reliability and changes with contraction. PM R 2014;6(3):227-34.

[14] Jeong JR, Ko YJ, Ha HG, et al. Reliability of rehabilitative ultrasonographic imaging for muscle thickness measurement of the rhomboid major. Clin Physiol Funct Imaging. 2016;36(2):134-8.

[15] Bentman S, O'Sullivan C, Stokes M. Thickness of the middle trapezius muscle measured by rehabilitative ultrasound imaging: description of the technique and reliability study. Clin Physiol Funct Imaging. 2010;30(6): 426-31.

[16] Ơsullivan C, Bentman S, Bennett K, et al. Rehabilitative ultrasound imaging of the lower trapezius muscle: technical description and reliability. J Orthop Sports Phys Ther. 2007;37(10):620-6.

[17] Temes W, Clifton AT, Hilton V, et al. Reliability and validity of thickness measurements of the supraspinatus muscle of the shoulder: an ultrasonography study. J Sport Rehabil. 2014;23(2):2013-23.

[18] Schneebeli A, Egloff M, Giampietro A, et al. Rehabilitative ultrasound imaging of the supraspinatus muscle: Intra-and interrater reliability of thickness and cross-sectional area. J Bodyw Mov Ther. 2014;18(2):266-72.

[19] Koppenhaver S, Harris D, Harris A, et al. The reliability of rehabilitative ultrasound imaging in the measurement of infraspinatus muscle function in the symptomatic and asymptomatic shoulders of patients with unilateral shoulder impingement syndrome. Int J Sports Phys Ther. 2015;10(2):128.

[20] Borstad JD. Measurement of pectoralis minor muscle length: validation and clinical application. J Orthop Sports Phys Ther. 2008;38(4):169-74. 
[21] Borstad JD. Resting position variables at the shoulder: evidence to support a posture-impairment association. Phys Ther. 2006;86(4):549-57.

[22] Koo TK, Li MY. A guideline of selecting and reporting intraclass correlation coefficients for reliability research. J Chiropr Med. 2016;15(2):155-63.

[23] Bland JM, Altman D. Statistical methods for assessing agreement between two methods of clinical measurement. Lancet. 1986;327(8476):307-10.

[24] Beckerman H, Roebroeck M, Lankhorst G, et al. Smallest real difference, a link between reproducibility and responsiveness. Qual Life Res. 2001;10(7):571-8.

[25] Koo TK, Wong C, Zheng Y. Reliability of sonomyography for pectoralis major thickness measurement. J Manipulative Physiol Ther. 2010;33(5):386-94.

[26] Lee S-J, Lim JY, Lee C-H, et al. Intra-and inter-rater reliability of muscle thickness measurement of the tibialis anterior using different inward pressures. J Phys Ther Sci. 2019;8(4):218-24.

[27] Kristjansson E. Reliability of ultrasonography for the cervical multifidus muscle in asymptomatic and symptomatic subjects. Man Ther. 2004;9(2):83-8. 